\title{
Rationalising empirical antibiotics for bloodstream infections: A retrospective study at a South African district-level hospital
}

\author{
A Engelbrecht, ${ }^{1} \mathrm{MB}$ ChB; C van der Westhuizen, ${ }^{2} \mathrm{MB} \mathrm{ChB} ; \mathrm{J} J$ Taljaard, ${ }^{3} \mathrm{MB} \mathrm{ChB}, \mathrm{DTM} \& \mathrm{H}$ \\ ${ }^{1}$ Division of General Medicine, Department of Medicine, Faculty of Medicine and Health Sciences, Stellenbosch University and Tygerberg \\ Hospital, Cape Town, South Africa \\ ${ }^{2}$ Division of Medical Microbiology, Department of Pathology, Stellenbosch University and National Health Laboratory Service, Cape Town, \\ South Africa \\ ${ }^{3}$ Division of Infectious Diseases, Department of Medicine, Faculty of Medicine and Health Sciences, Stellenbosch University and Tygerberg \\ Hospital, Cape Town, South Africa
}

Corresponding author: A Engelbrecht (amoriengelbrecht@gmail.com)

\begin{abstract}
Background. Incorrect empirical antibiotic therapy is one of the factors that contribute to poor clinical outcomes and the development of antimicrobial resistance. Knowledge of the local infectious disease burden and antibiotic resistance patterns can assist with development of strategies, updating of guidelines and subsequent improvement in initial empirical therapy.

Objectives. To determine whether the empirical antibiotic choice for treatment of septic episodes at a district-level hospital was appropriate according to national guidelines, and to describe the epidemiological features of the septic episode population being studied and depict their antibiotic susceptibility profile.

Methods. This was a retrospective, descriptive study of adult inpatients with bloodstream infections at Karl Bremer Hospital, Cape Town, South Africa. Laboratory and clinical data were obtained and analysed for the period 1 July 2017 - 30 June 2018. Septic episodes were subdivided into community-acquired bloodstream infection (CABSI) and hospital-acquired bloodstream infection (HABSI) study populations, and empirical antibiotics for both groups were assessed and compared with the adult Standard Treatment Guidelines and Essential Medicines List for South Africa, Hospital Level Care, 2015 edition (STG and EML).

Results. Our study sample consisted of 184 septic episodes, isolated from 176 patients. Nearly half of the septic episodes (49.5\%) were hospital acquired. Overall guideline adherence in the CABSI population was $88 \%$, compared with $58 \%$ in the HABSI population. The reasons for guideline non-adherence in the CABSI population were lack of source-appropriate empirical antibiotics $(n=7)$ and septic episodes where empirical antibiotics were indicated but not prescribed $(n=4)$, while in the HABSI group the main reason was that the patients were treated by community-acquired standards $(n=30 ; 33.0 \%)$. The in-hospital mortality rate for a septic episode in this study was $38 \%$. Considering the typical first-line antibiotics used, $77.3 \%$ of CABSIs were found to be susceptible to co-amoxiclav $(n=75)$ and $59.8 \%$ to ceftriaxone ( $n=58$ ). With the exclusion of methicillin-resistant Staphylococcus aureus and Acinetobacter baumannii isolates as confounders, HABSIs had a susceptibility of $86 \%$ to the piperacillin/tazobactam plus amikacin combination, $81 \%$ to ertapenem, $90 \%$ to imipenem and 93\% to meropenem.

Conclusions. This study demonstrates poor guideline adherence in HABSIs, emphasising the importance of distinguishing between CABSIs and HABSIs. The empirical antibiotics advised by the STG and EML were found to be appropriate in the majority of septic episodes. Future revision and improvement of prescribing practices can assist in rationalising empirical antibiotic decisions.
\end{abstract}

S Afr Med J 2022;112(1):27-33. https://doi.org/10.7196/SAMJ.2022.v112i1.15959

Bloodstream infection (BSI) remains a significant cause of morbidity and mortality worldwide. ${ }^{[1]}$ In 2017, an estimated 48.9 million cases of sepsis and 11 million sepsis-related deaths were reported globally, with in-hospital sepsis mortality in sub-Saharan Africa estimated at $\sim 19 \%{ }^{[1,2]}$ Ageing populations with more comorbidities, improved surveillance pertaining to sepsis, and a growing HIV pandemic all contribute to an increased incidence of sepsis. ${ }^{\left[{ }^{[3]}\right.}$ Determining the causative bacterial organism and its antibiotic susceptibility profile is of paramount importance for appropriate treatment. Antibiotic choices are often confounded by lack of knowledge regarding disease burden, local epidemiology and resistance patterns of the organisms involved.

Incorrect empirical antibiotic therapy is one of the factors that contribute to poor clinical outcomes and the development of antimicrobial resistance (AMR). Emphasis on empirical antibiotic therapy becomes important as, depending on local laboratory standard operating protocols, blood cultures can take up to a few days to identify the infective agent. Standardised antibiotic guidelines tailored to local susceptibility patterns are limited and frequently outdated. Currently, antimicrobial prescribing in the public sector in South Africa (SA) is guided by Standard Treatment Guidelines (STG) and is determined by the inclusion and availability of the medicine on the Essential Medicines List (EML). ${ }^{[4]}$ The ultimate choice of antibiotic prescribed is often the personal preference of the prescribing doctor. Knowledge of the local infectious disease burden and antibiotic resistance patterns can assist with development of strategies, updating of guidelines and subsequent improvement in initial empirical therapy. ${ }^{[5]}$ 
The importance of distinguishing between community-acquired BSI (CABSI) and hospital-acquired BSI (HABSI) has been well established, with increasing AMR demonstrated among healthcare-associated pathogens. ${ }^{[6]}$ With the emergence of carbapenem-resistant Enterobacterales (CRE), the empirical use of carbapenems for HABSIs has been a controversial issue. A number of carbapenem-sparing strategies have been proposed, and the combination of piperacillin/tazobactam plus amikacin is currently endorsed by the STG. Although the MERINO trial ${ }^{[7]}$ described a lower 30-day all-cause mortality with meropenem compared with piperacillin/tazobactam for extended-spectrum beta-lactamase (ESBL) BSI isolates, a meta-analysis consisting of 25 observational studies showed noninferiority of piperacillin/tazobactam compared with carbapenems. ${ }^{[8]}$ From an antimicrobial stewardship perspective, one could therefore argue that carbapenems should be reserved as empirical therapy for patients at high risk of ESBL infections, especially those with septic shock or poor source control. ${ }^{[9]}$

In the absence of reliable sepsis aetiology data, what constitutes 'appropriate' empirical antibiotic choices remains an open question in SA. ${ }^{[2]}$ Selecting appropriate empirical antibiotics is especially important in HABSI populations, where a relative increase in resistant organisms is suspected and the impact of empirical antibiotics can be profound. At a time when astute antibiotic stewardship is continually emphasised, periodic review of local antibiograms and prescription practices should be mandated.

\section{Objectives}

To determine whether the empirical antibiotic choices for treatment of septic episodes at a district-level hospital were appropriate according to national guidelines, to describe the epidemiological features of the septic episode population being studied, and to depict their antibiotic susceptibility profile (antibiograms).

\section{Methods \\ Study design}

A retrospective descriptive study of all adult ( $>13$ years) inpatients with BSIs at Karl Bremer Hospital (KBH), Cape Town, SA, between 1 July 2017 and 30 June 2018 was performed. Patients were subdivided into CABSI and HABSI study populations, and empirical antibiotics for both groups were assessed and compared with the current STG and EML at the time (Standard Treatment
Guidelines and Essential Medicines List for South Africa, Hospital Level Care, 2015 edition) ${ }^{[10]}$

\section{Setting}

$\mathrm{KBH}$ is a large district-level hospital in the Tygerberg Eastern Health District of the City of Cape Town Metropolitan Municipality. The hospital has a total of 310 beds, and treats an average of 11000 patients per month. Approximately 210 blood cultures are performed monthly among all adult patients. However, the impact of empirical antibiotics and the influence of blood cultures on antibiotic decision-making have not been assessed in recent times.

\section{Study population}

The study population was structured according to septic episodes. To achieve this, the laboratory information system of the National Health Laboratory Service (NHLS) was accessed to identify positive blood culture specimens during the period specified above. Only BSIs in adults (>13 years) were considered for the study. Clinical records and other microbiological reports were reviewed to determine whether a particular isolate was deemed a contaminant; these were excluded. Fungal isolates were also excluded. Lastly, to limit the data to a single septic episode of BSI, duplicate isolates were excluded (Fig. 1).

Our final study sample consisted of 184 septic episodes, isolated from 176 patients (8 patients had an additional septic episode during the study period). Furthermore, with the inclusion of multiple organisms from individual blood cultures, our dataset contained 199 pathogens (15 septic episodes had 2 organisms isolated). No septic episode was characterised as having $\geq 3$ organisms isolated.

\section{Data}

Demographics, clinical details, source of septic episode, antimicrobial management and patient outcome information were extracted from hospital and laboratory records for all patients who fitted the inclusion criteria and formed part of the study population. Antibiotic susceptibility results of identified organisms were obtained from the NHLS database. Additional study variables included duration of admission, departmental specialty responsible for the patient, ward admitted to and disposition of the patient. The appropriateness of empirical antibiotic choice was determined by adherence to the STG and EML.

All data and results were depersonalised of patient identifiers to ensure patient anonymity and to limit reporting bias. All anonymised data collected were captured onto a secure REDCap online database (Vanderbilt University, USA).

\section{Study parameters}

BSIs are infectious diseases defined by the presence of viable bacterial or other pathogenic microbes in the bloodstream. For the purpose of this study, only bacterial isolates were included. A septic episode encompasses each BSI as identified by a single positive blood culture that isolates one or more pathogens.

CABSIs are BSIs that occur in outpatients or are first identified $<48$ hours after admission to hospital. HABSIs are defined as infections developing at least 48 hours after admission or within 3 months after discharge from a healthcare facility. ${ }^{[1]}$ As such, they encompass hospital-acquired BSI. The distinction necessarily influences the epidemiology and susceptibility of the organisms involved, and ultimately the choice of empirical antibiotic treatment.

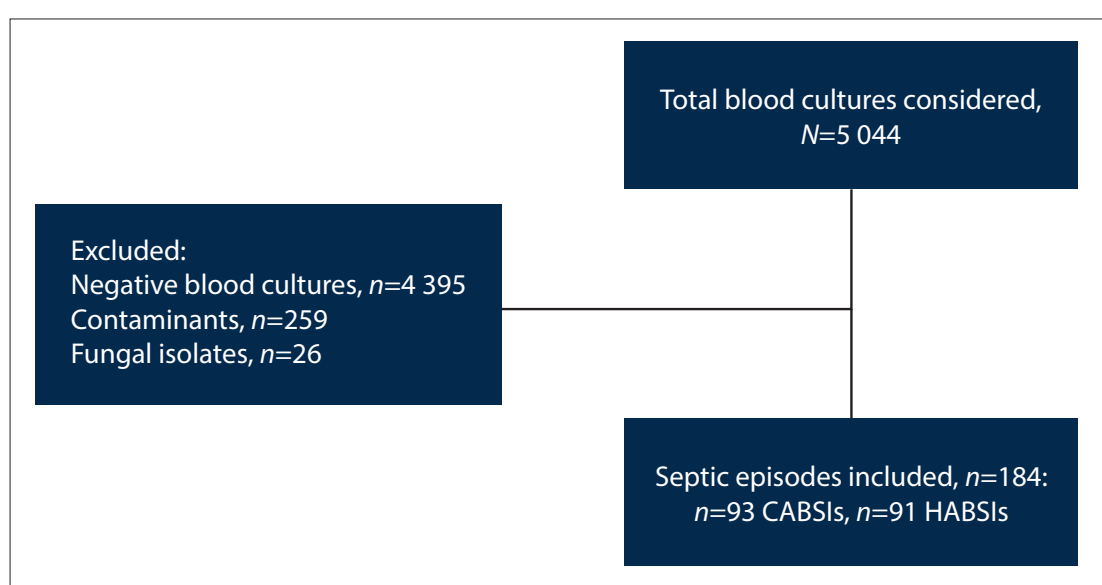

Fig. 1. Study population. $(C A B S I$ = community-acquired bloodstream infections; HABSIs = hospitalacquired bloodstream infections.) 
Contaminants were regarded as blood cultures that were positive for growth due to organisms that were not truly present in the bloodstream, which when identified were not in keeping with the clinical features of the case, further supported by the treating clinician's clinical assessment and decision not to provide therapy targeted to the isolate. ${ }^{[12]}$

Duplicate isolates were defined as positive blood culture(s) from the same patient where an identical pathogen was isolated with an indistinguishable susceptibility profile within 2 weeks of the index septic episode, excluding the initial isolate. ${ }^{[13]}$

Appropriate empirical antibiotic therapy was defined as administration of an antimicrobial agent as stipulated in the current STG and EML. Note that considerations regarding dosages, dosing intervals, route of administration and treatment duration did not form part of the definition. This was because the study focused on appropriate choice rather than use of antibiotics, and rationalising the decision within the empirical context.

The 4th edition of the adult Standard Treatment Guidelines and Essential Medicines List for South Africa, Hospital Level Care (2015) ${ }^{[10]}$ was used to evaluate prescription practices in this study, as it was the most recently updated edition at the time.

\section{Statistical analysis}

The level of statistical significance was set at $p<0.05$. We used descriptive methods to assess the distribution of BSIs and their antibiograms. Antibiotic susceptibility was recorded as susceptible or resistant. For the sake of simplicity, isolates with intermediate breakpoints were also categorically regarded as resistant.

\section{Ethical considerations and regulatory compliance}

Ethical approval for this study was obtained from the Health Research Ethics Committee of Stellenbosch University (ref. no. S20/01/004). Provincial approval was obtained from the Western Cape Provincial Health Impact Assessment Committee. The study was conducted in accordance with the Declaration of Helsinki 2013 and the SA National Department of Health Guidelines for Good Clinical Practice in the Conduct of Clinical Trials. ${ }^{[14]}$

\section{Results \\ Patient demographics}

Of the 176 patients included in the study, 87 (49.4\%) were male and $89(50.6 \%)$ were female. The mean (standard deviation) age of patients was 51 (19) years (minimum 15, maximum 90) (Table 1). Clinical information with regard to comorbidities was missing for 3 patients. Among the 173 patients with available clinical information, hypertension was the most common comorbidity, present in $35.8 \%$ of patients. A total of 58 patients (33.5\%) were people living with HIV, of whom only 15 (25.9\%) were on antiretroviral therapy. The in-hospital mortality rate for a septic episode in this study was $38 \%$. The proportion of CABSI patients who died (38.6\%) was significantly lower $(p<0.05)$ than the proportion of HABSI patients who died (61.4\%). A Pearson $\chi^{2}$ coefficient of 5.19 was found.

\section{Pathogen distribution}

The presumed source of a septic episode was determined by the clinical evaluation of the attending clinician. The most common source for bloodstream isolates was lower respiratory tract infections. This was followed by urinary tract infections and then skin and soft-tissue infections (mainly comprising cellulitis, wet gangrene, necrotising fasciitis and abscesses) (Table 2).

Escherichia coli was the most frequently isolated pathogen ( $n=42 ; 21.1 \%)$, followed by Staphylococcus aureus $(n=38 ; 19.1 \%)$

\begin{tabular}{ll} 
Table 1. Patient characteristics & \\
\hline Demographics $(N=176)$ & \\
Age (years), mean (SD) & $51(19)$ \\
Male sex, $n(\%)$ & $87(49.4)$ \\
Comorbid conditions ${ }^{\star}(N=173), n(\%)$ & $62(35.8)$ \\
Hypertension & $58(33.5)$ \\
HIV-positive & $41(23.7)$ \\
Diabetes & $23(13.3)$ \\
Smoking & $16(9.1)$ \\
Alcohol abuse & $13(7.4)$ \\
Active malignancy & \\
${ }^{*}$ Three patients excluded owing to unavailable data. &
\end{tabular}

and Klebsiella pneumoniae ( $n=25 ; 12.6 \%)$. Among CABSI isolates, E. coli $(n=22 ; 22.7 \%)$, followed by S. aureus $(n=19 ; 19.6 \%)$ and Streptococcus pneumoniae $(n=13 ; 13.4 \%)$, predominated. Ninetyone $(49.5 \%)$ of the septic episodes were HABSIs. Three cases of community-acquired (CA) Pseudomonas aeruginosa were identified; in 1 of these, $P$. aeruginosa was also cultured on an intrauterine contraceptive device, while the other 2 patients had evidence of underlying structural lung disease. CA Stenotrophomonas maltophilia was isolated in 1 patient with end-stage renal failure secondary to HIV-associated nephropathy, who had a history of frequent exposure to the healthcare system during the preceding months.

Gram-negative bacilli constituted the largest group of HABSIs, among which the Enterobacterales group predominated $(n=49$; 48.0\%). Non-fermentative Gram-negative bacilli comprised $13.7 \%$ of this subpopulation. The most commonly isolated hospital-acquired (HA) organisms were E. coli $(n=20 ; 19.6 \%), K$. pneumoniae $(n=20$; $19.6 \%)$ and S. aureus $(n=19 ; 18.6 \%)$ (Table 3$)$.

\section{Antibiotic susceptibility: CABSIs}

Overall, the carbapenems $(n=96 ; 99.0 \%)$ followed by the aminoglycosides $(n=95 ; 98.0 \%)$ were the antibiotic classes that displayed the highest susceptibility among CABSIs, while 52.6\% of CABSIs ( $n=51$ ) displayed resistance to amoxicillin/ampicillin (Fig. 2). Considering the typical first-line antibiotics used in casualties/ emergency departments, $77.3 \%$ of CABSIs were found to be susceptible to co-amoxiclav $(n=75)$ and $59.8 \%$ to ceftriaxone $(n=58)$. A notable finding to explain these seemingly low susceptibilities was the considerable number of AmpC beta-lactamase producers and non-fermentative Gram-negative bacilli.

CA Enterobacterales were predominantly susceptible to ceftriaxone $(n=33 ; 82.5 \%)$, gentamicin $(n=33 ; 82.5 \%)$ and ciprofloxacin $(n=32$; $80.0 \%$ ) (Fig. 3). ESBL production occurred in 5 isolates (12.5\%), of which 4 were reported susceptible to piperacillin/tazobactam (presumed low mean inhibitory concentrations) and none was resistant to gentamicin or any of the carbapenems.

All of the CA S. aureus $(n=19)$ were methicillin-sensitive, and 18 of these remained susceptible to clindamycin and all to co-trimoxazole. All S. pneumoniae isolates $(n=13)$ were susceptible to ceftriaxone, whereas only 1 was reported as resistant to amoxicillin.

\section{Antibiotic susceptibility: HABSIs}

HABSIs overall had a susceptibility of $77 \%$ to the piperacillin/ tazobactam plus amikacin combination, $66 \%$ to ertapenem, $77 \%$ to imipenem and $79 \%$ to meropenem. When methicillin-resistant S. aureus (MRSA) and Acinetobacter baumannii isolates are excluded as confounders, these susceptibility numbers recalculate to $86 \%$ to 


\begin{tabular}{|c|c|}
\hline Clinical source ( $N=184$ episodes $)$ & Organism isolated ( $N=199$ organisms), $n(\%)$ \\
\hline \multirow[t]{5}{*}{ Pneumonia $(N=51)$} & Staphylococcus aureus, 15 (7.5) \\
\hline & Streptococcus pneumoniae, 11 (5.5) \\
\hline & Klebsiella pneumoniae, 7 (3.5) \\
\hline & Acinetobacter baumannii, 6 (3.0) \\
\hline & Other, 17 (8.5) \\
\hline \multirow[t]{4}{*}{ Urinary tract infection $(N=47)$} & Escherichia coli, 25 (12.6) \\
\hline & K. pneumoniae, 8 (4.0) \\
\hline & S. aureus, 5 (2.5) \\
\hline & Other, 13 (6.5) \\
\hline \multirow[t]{4}{*}{ Skin, soft tissue and wound $(N=31)$} & S. aureus, 7 (3.5) \\
\hline & E. coli, $5(2.5)$ \\
\hline & K. pneumoniae, 3 (1.5) \\
\hline & Other, 19 (9.5) \\
\hline \multirow[t]{4}{*}{ Intra-abdominal sepsis $(N=17)$} & E. coli, $6(3.0)$ \\
\hline & Salmonella typhi, 4 (2.0) \\
\hline & K. pneumoniae, 3 (1.5) \\
\hline & Other, 7 (3.5) \\
\hline \multirow[t]{3}{*}{ Pelvic (gynaecological) sepsis $(N=4)$} & E. coli, $2(1.0)$ \\
\hline & K. pneumoniae, $1(0.5)$ \\
\hline & Pseudomonas aeruginosa, 1 (0.5) \\
\hline \multirow{2}{*}{ Meningitis $(N=4)$} & S. pneumoniae, 2 (1.0) \\
\hline & Listeria monocytogenes, 2 (1.0) \\
\hline \multirow[t]{2}{*}{ Infective endocarditis $(N=3)$} & S. aureus, $2(1.0)$ \\
\hline & Streptococcus sanguinus, 1 (0.5) \\
\hline \multirow[t]{2}{*}{ Joint and bone $(N=3)$} & S. aureus, $2(1.0)$ \\
\hline & Non-typhoidal Salmonella, 1 (0.5) \\
\hline Intravascular catheter infection $(N=2)$ & S. aureus, $2(1.0)$ \\
\hline \multirow[t]{4}{*}{ Unknown $(N=22)$} & S. aureus, 5 (2.5) \\
\hline & Streptococcus pyogenes, 4 (2.0) \\
\hline & K. pneumoniae, 3 (1.5) \\
\hline & Other, $10(5.0)$ \\
\hline
\end{tabular}

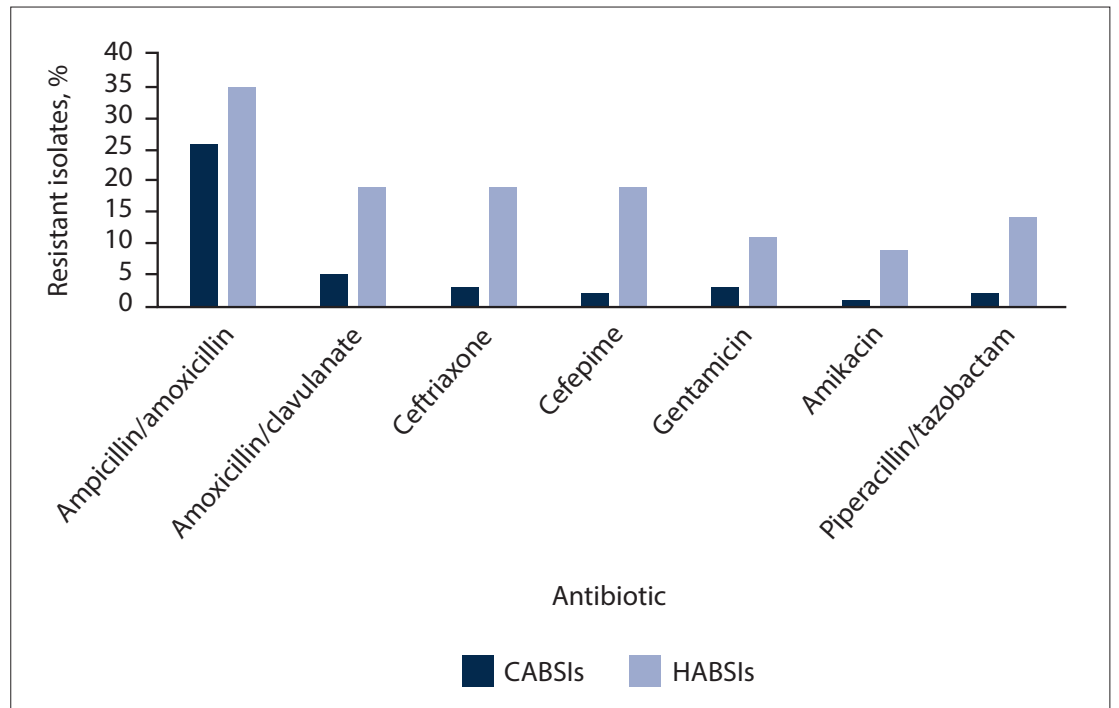

Fig. 2. Antimicrobial resistance of bloodstream isolates. (CABSIs = community-acquired bloodstream infections; HABSIs = hospital-acquired bloodstream infections.)

the piperacillin/tazobactam plus amikacin combination, $81 \%$ to ertapenem, $90 \%$ to imipenem and $93 \%$ to meropenem.

Of the HA Enterobacterales, $36.7 \%(n=18)$ were susceptible to ceftriaxone, $63.3 \%(n=31)$ to piperacillin/tazobactam and $79.6 \%(n=39)$ to amikacin. In keeping with the ceftriaxone susceptibility/resistance, the ESBL Enterobacterales (ESBL-E) rate was $63.3 \%$ $(n=31)$, with resistance patterns similar for ceftriaxone and cefepime. Of these ESBL-E, $83.7 \%$ were reported to be susceptible to the combination of piperacillin/tazobactam plus amikacin. One CRE was identified that only demonstrated susceptibility to co-trimoxazole and tigecycline.

HA S. aureus was found to be methicillin sensitive in $57.9 \%$ of cases, while $84.2 \%$ remained susceptible to clindamycin. No vancomycin resistance was detected among these isolates.

HA P. aeruginosa had $100 \%$ susceptibility to ciprofloxacin and gentamicin, and $75.0 \%$ susceptibility to piperacillin/tazobactam.

A. baumannii had a high prevalence of multidrug resistance, with gentamicin being reported as the antibiotic with the most consistent susceptibility at $77.8 \%$.

\section{Antibiotic adherence: CABSIs}

Ceftriaxone $(n=49 ; 52.7 \%)$ and co-amoxiclav 


\begin{tabular}{|c|c|c|}
\hline Pathogens & CABSIs $(N=97 ; 48.7 \%), n(\%) / n$ & HABSIs $(N=102 ; 51.3 \%), n(\%) / n$ \\
\hline Gram-negative & $49(24.6)$ & $68(34.2)$ \\
\hline \multicolumn{3}{|l|}{ Enterobacterales } \\
\hline Escherichia coli & 22 & 20 \\
\hline Klebsiella pneumoniae & 5 & 20 \\
\hline Proteus mirabilis & 5 & 2 \\
\hline Serratia marcescens & 1 & 3 \\
\hline Salmonella typhi & 4 & - \\
\hline Non-typhoidal Salmonella & 1 & 1 \\
\hline Other genus/species & 2 & 3 \\
\hline \multicolumn{3}{|l|}{ Non-fermenters } \\
\hline Acinetobacter baumannii & 0 & 9 \\
\hline Pseudomonas aeruginosa & 3 & 4 \\
\hline Stenotrophomonas maltophilia & 1 & 1 \\
\hline Other & 5 & 5 \\
\hline Gram-positive & $48(24.1)$ & $34(32.1)$ \\
\hline Staphylococcus aureus & 19 & 19 \\
\hline \multicolumn{3}{|l|}{ Streptococci } \\
\hline Streptococcus pneumoniae & 13 & 1 \\
\hline Streptococcus pyogenes & 5 & 2 \\
\hline Other species & 4 & 0 \\
\hline \multicolumn{3}{|l|}{ Enterococci } \\
\hline Enterococcus faecalis & 2 & 5 \\
\hline Enterococcus faecium & 1 & 2 \\
\hline Other species & 1 & 1 \\
\hline Listeria monocytogenes & 2 & 0 \\
\hline Other & 1 & 4 \\
\hline
\end{tabular}

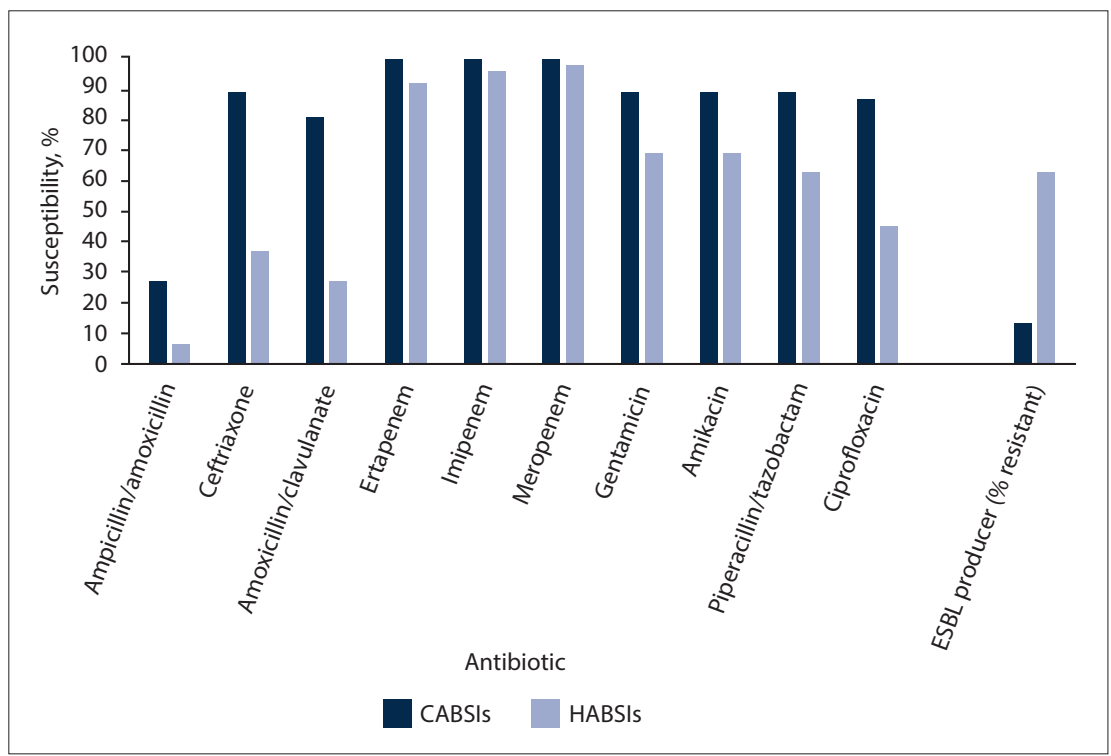

Fig. 3. Enterobacterales. (ESBL = extended-spectrum beta-lactamase. $)$

$(n=29 ; 31.2 \%)$ were the most frequently prescribed empirical antibiotics for CABSI episodes. Adherence to the STG and EML for CABSI prescription was calculated at $88 \%$. The reasons for inappropriate empirical antibiotics (i.e. non-adherence to the STG and EML) were the lack of source- appropriate empirical antibiotics $(n=7)$ and septic episodes where empirical antibiotics were indicated but not prescribed $(n=4)$.

The study found that $89.2 \%$ of empirical antibiotics prescribed in CABSIs had an indication to be changed to a definitive treatment agent after a blood culture result became available. These indications included escalation to broaden cover $(n=4 ; 4.3 \%)$, de-escalation to narrower spectrum $(n=51$; $54.8 \%$ ), a change to a different class of antibiotic ( $n=24 ; 25.8 \%)$, or to initiate therapy in cases where no empirical antibiotics were prescribed ( $n=4 ; 4.3 \%$ ) (Fig. 4). However, only $40.9 \%$ of these empirical antibiotics were actually changed. This seemingly low percentage is confounded by patients who died or were transferred to tertiary care before blood culture results were reported. Actual changes of antibiotic therapy that occurred included escalation to broadspectrum cover $(n=4 ; 4.3 \%)$, de-escalation to narrower spectrum $(n=20 ; 21.5 \%)$ and changes to a different class of antibiotic $(n=14 ; 15.1 \%)$.

\section{Antibiotic adherence: HABSIs}

The combination of piperacillin/tazobactam plus amikacin was the most common empirical antibiotic therapy prescribed for HABSIs $(n=23 ; 25.3 \%)$, followed by ceftriaxone $(n=20 ; 22.0 \%)$ and co-amoxiclav $(n=20 ; 22.0 \%)$.

Forty-two percent $(n=38)$ of empirical antibiotics prescribed in HABSIs were not in accordance with the STG and EML. This can 
be attributed to the large number of HABSIs that were inappropriately treated as a CABSI $(n=30 ; 33.0 \%)$. Other causes for nonadherence included the lack of empirical antibiotics prescribed when indicated $(n=5$; $5.5 \%)$ and the lack of source-appropriate empirical antibiotics $(n=3 ; 3.3 \%)$.

The study found that $74.7 \%$ of empirical antibiotics prescribed for HABSIs had an indication to be changed after a blood culture result became available. These indications included escalation to broader spectrum $(n=22 ; 24.2 \%)$, de-escalation to narrower spectrum $(n=25 ; 27.5 \%)$, change to a different class of antibiotic $(n=17$; $18.7 \%)$ and to initiate therapy in cases where no empirical antibiotics were prescribed $(n=4 ; 4.4 \%)$ (Fig. 5). Forty-three percent of empirical antibiotics were subsequently changed. Once again, the confounders here were patients who died or were transferred to tertiary care before blood culture results were reported. Actual changes of antibiotic therapy that occurred included escalation to broad-spectrum cover $(n=19 ; 20.9 \%)$, de-escalation to narrower spectrum $(n=6$; $6.6 \%)$ and changes to a different class of antibiotic $(n=14 ; 15.4 \%)$.

\section{Guideline susceptibility comparison} Empirical antibiotics as stipulated by the STG and EML for a particular source of infection were directly compared with the antibiograms of the septic episodes. The study calculated a $75.0 \% \quad(n=138)$ susceptibility agreement. Crudely put, following the STG and EML would have ensured that $75.0 \%$ of the septic episodes in this study were appropriately covered with empirical antibiotics.

Explanations for disagreement with the antibiograms included the presence of resistant isolates $(n=27 ; 14.7 \%)$ and inappropriate $S$. aureus cover $(n=19 ; 10.3 \%)$. Although certain antibiotics, e.g. ceftriaxone and co-amoxiclav, may be regarded as reasonable empirical cover (although not definitive) for $S$. aureus, they were not included in the antibiogram reports. As such, they were not deemed appropriate according to the antibiograms. Excluding $S$. aureus septic episodes would have increased the correlation of the STG and EML with the antibiograms to $83.6 \%$.

\section{Discussion}

The contamination rate of blood cultures found in this study was close to $40 \%$, which is comparable with a similar study at Groote Schuur Hospital, Cape Town. ${ }^{[6]}$ This relatively high percentage may represent

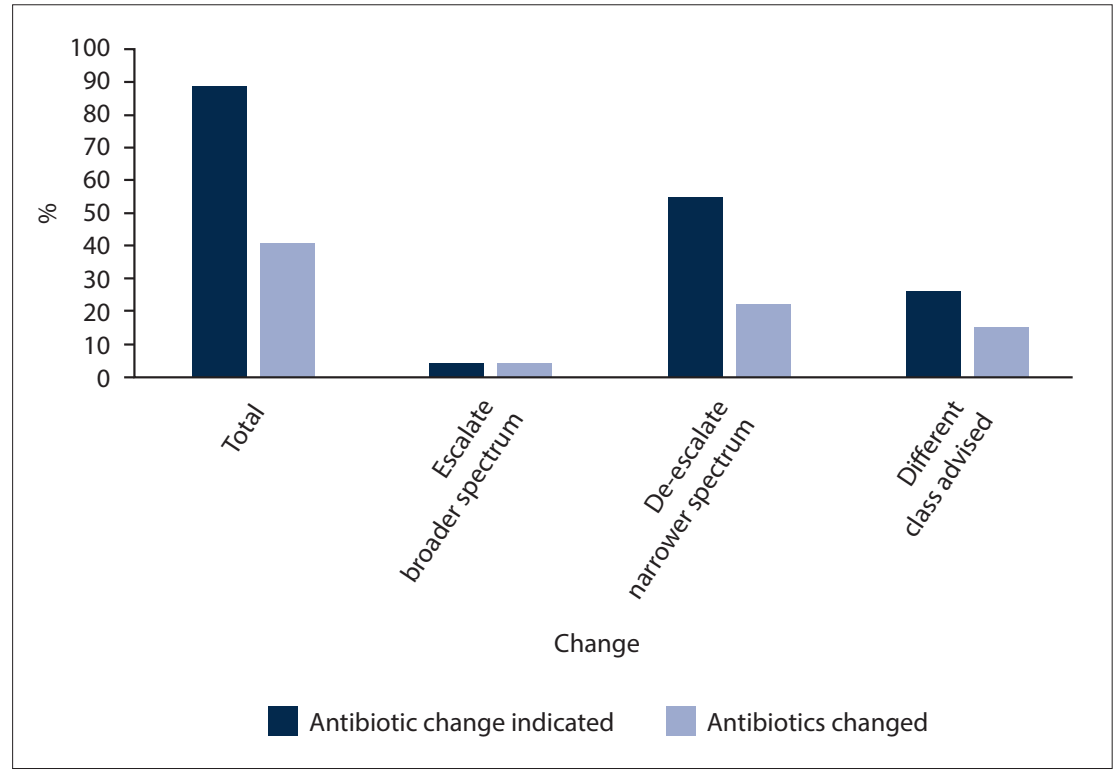

Fig. 4. Antibiotic therapy changes in CABSIs. (CABSIs = community-acquired bloodstream infections.)

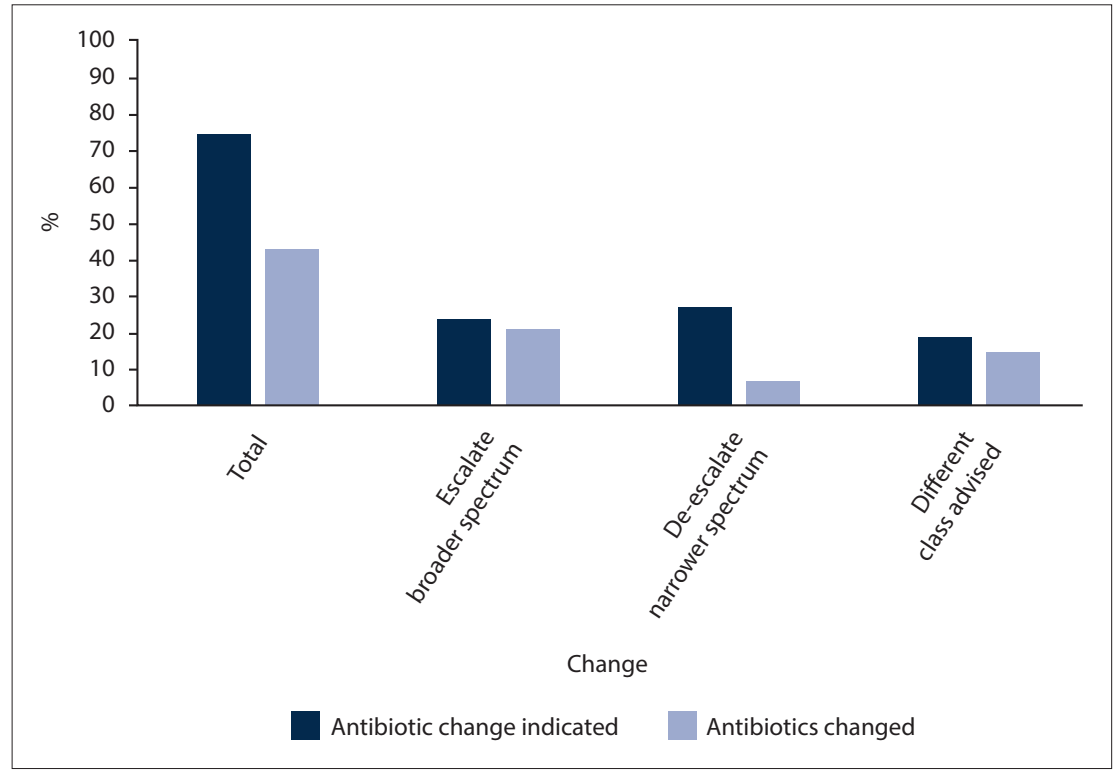

Fig. 5. Antibiotic therapy changes in HABSIs. (HABSIs = hospital-acquired bloodstream infections.)

true contaminants, but could also include isolates for which clinical information was lacking. This finding can also partially be explained by the majority of blood cultures being taken while patients were still in the emergency department, where overcrowding and time constraints often lead to lower compliance with correct sampling technique. However, it is evident that education for all healthcare providers involved in blood culture collection is necessary. Coagulasenegative staphylococci accounted for $76 \%$ of these contaminants.

The overall in-hospital mortality rate for septic episodes in this study was $38 \%$. This figure increased to $46 \%$ in patients who did not receive appropriate empirical antibiotics, confirming that survival improves when empirical antibiotic therapy is given in accordance with the STG and EML.

The most prevalent organisms isolated in this study for CABSIs (E. coli, S. aureus and S. pneumoniae) and HABSIs (K. pneumoniae, E. coli, S. aureus and Acinetobacter species) were generally in keeping with other recent studies. ${ }^{[6,15]}$ The predominance of Gram-negative organisms among HABSIs was also evident, making the distinction between CA and HA infection imperative. ESBLs still represent an important AMR mechanism, outlined by the production of ESBL occurring in two-thirds of all HA Enterobacterales. This high occurrence re-emphasises the need for stringent 
infection prevention control measures in patients with HABSIs to limit the spread of transferable resistance in the healthcare setting. The notable presence of $S$. aureus found in this study should prompt consideration for directed empirical antibiotics in suspect cases, e.g. skin and soft-tissue infection, infective endocarditis, septic arthritis and undifferentiated sepsis, as well as non-resolving pneumonia. CA S. aureus remained largely methicillin sensitive, while 39\% of HA S. aureus were methicillin resistant, advocating for the use of empirical vancomycin for indicated presentations. All cases of blood culture-positive endocarditis were caused by methicillinsusceptible S. aureus. As expected, A. baumannii isolates showed low susceptibility rates to most antibiotics.

During the national listeria outbreak, Listeria monocytogenes comprised $40 \%$ of all cases of blood culture-positive meningitis, emphasising the diagnostic value of blood cultures in high-risk groups, especially in cases where lumbar puncture is contraindicated.

Carbapenems are frequently considered the drugs of choice against ESBL-E, but predicting ESBL-E as the cause of a HABSI presents clinical difficulty. In this study, we were unable to demonstrate that empirical therapy with carbapenems in patients with HABSIs was superior to the combination of piperacillin/tazobactam plus amikacin. This encourages the use of the combination as a carbapenem-sparing strategy. Meropenem was found to be collectively effective in almost all bloodstream isolates in this study, except in the case of one CRE, MRSA and most A. baumannii isolates.

Nearly half of the patients with HABSIs received inappropriate empirical antibiotics according to the STG and EML, and $42 \%$ of all empirical antibiotics were changed after the blood culture result became available. This high percentage emphasises the fact that blood cultures remain an important diagnostic modality that actively contributes to therapeutic decision-making. The STG and EML were well adhered to in CABSIs. In the HABSI group, the most common cause for inappropriate empirical antibiotics was the fact that these infections were treated by CA standards. The importance of obtaining a careful history with regard to recent exposure to the healthcare system, and risk stratifying accordingly, cannot be overstated.

\section{Study limitations}

There are limitations to this study. Firstly, it was a time-specific, single-centre retrospective study. However, the paucity of local data makes the epidemiological findings described of value and importance. Secondly, although much consideration was applied, blood cultures deemed to be contaminants may in fact have harboured true pathogens. These would have been excluded from the study. Albeit anecdotal, it is not believed that this would skew results in a consequential fashion. Thirdly, owing to the retrospective nature of this study, long-term outcomes and estimates of morbidity were not reported on.

In view of the above, and also the inherent design of such a study, the findings should be extrapolated with caution.

\section{Conclusions}

This study emphasised the importance of distinguishing between CABSIs and HABSIs, and the subsequent impact thereof on empirical antibiotic choices. The empirical antibiotics advised by the STG and EML, in conjunction with individualised clinical evaluation, were found to be appropriate in the majority of septic episodes in the study. CABSI isolates preserve significant susceptibility to commonly used first-line antibiotics, advocating for the initial use of narrow-spectrum empirical antibiotics. The empirical combination of piperacillin/tazobactam plus amikacin was found to be an effective carbapenem-sparing strategy in HABSIs.

Although this was a time-specific, single-centre retrospective study, it provides sufficient evidence that routine evaluation of susceptibility profiles of BSI culture results, as well as revision of prescribing practices, can assist in rationalising empirical antibiotic decisions. Knowledge of local antibiograms and correct prescription practices will improve appropriate empirical treatment for individual patients and ultimately improve outcome in patients with BSIs.

Declaration. The research for this study was done in partial fulfilment of the requirements for AE's MMed (Internal Medicine) degree at Stellenbosch University.

Acknowledgements. Special thanks to Dr Amy Slogrove for her guidance with research methodology. We thank Dr Moleen Dzikiti for assisting with the data analysis and her subsequent interpretation of the statistical outcomes. We also thank the staff at Karl Bremer Hospital for facilitating data collection.

Author contributions. AE: primary author, overall conception and design of the study, acquisition of data at Karl Bremer Hospital, interpretation, drafting and critical revising of the work; CvdW: assisted in acquisition of data at Karl Bremer Hospital, interpretation and drafting of work; JJT: supervisor, interpretation, and critical revising of the work. All authors read and approved the final manuscript.

Funding. None.

Conflicts of interest. None.

1. Rudd KE, Johnson SC, Agesa KM, et al. Global, regional, and national sepsis incidence and mortality 1990 - 2017: Analysis for the Global Burden of Disease Study. Lancet 2020;395(10219):200-211. https:// 1990 - 2017: Analysis for the Global Burc
doi.org/10.1016/s0140-6736(19)32989-7

2. Lewis JM, Feasey NA, Rylance J. Aetiology and outcomes of sepsis in adults in sub-Saharan Africa: A systematic review and meta-analysis. Crit Care 2019;23(1):212. https://doi.org/10.1186/s13054-0192501-y

3. Singer M, Deutschman CS, Seymour C, et al. The third international consensus definitions for sepsis and septic shock (sepsis-3). JAMA 2016;315(8):801-810. https://doi.org/10.1001/jama.2016.0287

4. Perumal-Pillay VA, Suleman F. Selection of essential medicines for South Africa: An analysis of indepth interviews with national Essential Medicines List committee members. BMC Health Serv Res 2017;17(1):17. https://doi.org/10.1186/s12913-016-1946-9

5. Laupland KB, Church DL. Population-based epidemiology and microbiology of community-onset bloodstream infections. Clin Microbiol Rev 2014;27(4):647-664. https://doi.org/10.1128/cmr.00002-14

bloodstream infections. Clin Microbiol Rev 2014;27(4):647-664. https://doi.org/10.1128/cmr.00002-14

McKay R, Bamford C. Community- versus healthcare-acquired bloodstream infections at Groote Schuur
Hospital, Cape Town, South Africa. S Afr Med J 2015:105 (5):363-369. https://doi.org/10.7196/SAMJ.8183 Hospital, Cape Town, South Africa. S Afr Med J 2015;105(5):363-369. https://doi.org/10.7196/SAMJ.8183 Harris PNA, Tambyah PA, Lye DC, et al. Effect of piperacillin-tazobactam vs meropenem on 30-day mortality for patients with $E$ coli or Klebsiella pneumoniae bloodstream infection and ceftriaxone resistance: A randomised clinical trial. JAMA 2018;320(10):984-994. https://doi.org/10.1001/ jama.2018.12163

8. Sfeir MM, Askin G, Christos P. Beta-lactam/beta-lactamase inhibitors versus carbapenem for bloodstream infections due to extended-spectrum beta-lactamase-producing Enterobacteriaceae Systematic review and meta-analysis. Int J Antimicrob Agents 2018;52(5):554-570. https://doi. org/10.1016/j.ijantimicag.2018.07.021

9. Sfeir MM. Post-MERINO trial: Any role for piperacillin-tazobactam in treating bloodstream infections caused by extended-spectrum beta-lactamase producing Enterobacteriaceae? Int J Antimicrob Agent 2019;53(5):557-558. https://doi.org/10.1016/j.jiantimicag.2019.03.007

10. National Department of Health, South Africa. Standard Treatment Guidelines and Essential Medicines List for South Africa: Hospital level adults. 4th ed. Pretoria: NDoH, 2015. https://www.samedical.org/ cmsuploader/viewFile/447 (accessed 3 November 2021).

11. Friedman ND, Kaye KS, Stout JE, et al. Healthcare-associated bloodstream infections in adults: A reason to change the accepted definition of community-acquired infections. Ann Intern Med 2002;137(10):791to change the accepted definition of community-acquired infection
797. https://doi.org/10.7326/0003-4819-137-10-200211190-00007

12. Hall KK, Lyman JA. Updated review of blood culture contamination. Clin Microbiol Rev 2006;19(4):788Hall KK, Lyman JA. Updated review of blood
802. https://doi.org/10.1128/cmr.00062-05

13. Magee JT. Effects of duplicate and screening isolates on surveillance of community and hospital antibiotic resistance. J Antimicrob Chemother 2004;54(1):155-162. https://doi.org/10.1093/jac/dkh295

14. National Department of Health, South Africa. South African Good Clinical Practice: Clinical Trial Guidelines. Pretoria: NDoH, 2020. https://www.sahpra.org.za/wp-content/uploads/2021/06/SAGCP-2020_Final.pdf (accessed 3 November 2021).

15. Anderson DJ, Moehring RW, Sloane R, et al. Bloodstream infections in community hospitals in the 21st century: A multicenter cohort study. PLoS ONE 2014;9(3):e91713. https://doi.org/10.1371/journal. pone. 0091713

Accepted 12 August 2021 